\title{
Efficacy and Renal Safety of Dapagliflozin in Patients with Type 2 Diabetes Mellitus Also Receiving Metformin: A Real-Life Experience
}

\author{
Alessandro Scorsone $\mathbb{D}^{1},{ }^{1}$ Gabriella Saura, ${ }^{1}$ Mattia Fleres, ${ }^{1}$ Lucia Spano, ${ }^{1}$ Vito Aiello, ${ }^{1}$ \\ Davide Brancato, ${ }^{1}$ Anna Di Noto, ${ }^{1}$ Francesca Provenzano, ${ }^{2}$ and Vincenzo Provenzanoo ${ }^{1}$ \\ ${ }^{1}$ Regional Referral Centre for Insulin Pump Implantation and Diabetes, Civic Hospital, Partinico, Palermo, Italy \\ ${ }^{2}$ Department of Internal Medicine, University of Palermo, Palermo, Italy \\ Correspondence should be addressed to Alessandro Scorsone; alexscorsone@gmail.com
}

Received 21 September 2017; Revised 5 March 2018; Accepted 13 March 2018; Published 3 May 2018

Academic Editor: Bernard Portha

Copyright (C) 2018 Alessandro Scorsone et al. This is an open access article distributed under the Creative Commons Attribution License, which permits unrestricted use, distribution, and reproduction in any medium, provided the original work is properly cited.

\begin{abstract}
Introduction. This study aimed at evaluating the efficacy and safety of dapagliflozin in patients with type 2 diabetes (T2D) who also received metformin in clinical practice in Italy. Methods. This was a retrospective observational study and it included data from patients who received dapagliflozin $10 \mathrm{mg}$ once daily in conjunction with metformin for 12 months (DAPA + MET). In those with inadequate glycemic control, insulin or glimepiride was added after 30 days (DAPA + MET + other glucose-lowering drugs). Efficacy assessments included glycosylated hemoglobin $\left(\mathrm{HbA}_{1 \mathrm{c}}\right)$ levels at 6 and 12 months, as well as body mass index (BMI) and lipid parameters at 12 months. Safety was also assessed. Results. Data on 66 patients were included. In both groups, $\mathrm{HbA}_{1 \mathrm{c}}$ was significantly reduced at 6 and 12 months compared with baseline and significant reductions in $\mathrm{HbA}_{1 \mathrm{c}}$ were observed at 12 months compared with 6 months. Over the 12-month treatment period, dapagliflozin significantly reduced BMI in both groups. No significant changes in lipid parameters were observed in either group and no detrimental effects on renal function were detected. Conclusions. Dapagliflozin is effective and safe in patients with T2D also receiving metformin. Glycemic control was already achieved with dapagliflozin + metformin, and add-on therapy was not associated with further improvements.
\end{abstract}

\section{Introduction}

Over the past 20 years, the proportion of Italians with diabetes in the general population increased from $3.4 \%$ to $5.5 \%$ and the vast majority (91\%) have type 2 diabetes. In Italy, the management of diabetes is associated with approximately $€ 10$ billion per year in direct and indirect costs [1].

The principal goal of effective treatment of type 2 diabetes is to reduce blood glucose [2]. At the same time, because type 2 diabetes is characterized by systemic dysregulation of metabolism and is strongly associated with obesity [3], glucose-lowering agents that reduce body weight are preferable to those that have no effect on or increase it. Cardiovascular diseases, for which obesity is a major risk factor, are estimated to cause $40 \%$ of all deaths attributed to type 2 diabetes [4].
Dapagliflozin is a glucose-lowering agent that acts by inhibiting sodium glucose cotransporter 2 (SGLT2). Located in the proximal tubule of the nephron, SGLT2 is responsible for the reabsorption of most of the previously filtered glucose [5]. SGLT2 inhibition results in glycosuria and represents an insulin-independent method of reducing blood glucose levels. It also results in a reduction in body weight due to the loss of calories contained in excreted glucose [6]. Studies have demonstrated that dapagliflozin reduced serum glycosylated hemoglobin $\left(\mathrm{HbA}_{1 \mathrm{c}}\right)$ levels and body weight in patients with type 2 diabetes when used as monotherapy [7], as well as in combination with other glucose-lowering agents [8-11].

The aim of this study was to evaluate the efficacy and safety of dapagliflozin in patients with type 2 diabetes also receiving metformin in clinical practice in Italy. 


\section{Methods}

2.1. Study Design. This retrospective observational study investigated the effectiveness and renal safety of dapagliflozin $10 \mathrm{mg}$ once daily in adults with type 2 diabetes mellitus who were also receiving metformin 1.5-2.5 g/day (DAPA + MET). Data from outpatients treated at the Regional Referral Centre for Insulin Pump Implantation and Diabetes at the Civic Hospital of Palermo, Italy, who initiated dapagliflozin treatment between March 2015 and March 2016 and who had undergone a follow-up visit at 12 months were included in the analysis. We included 66/94 subjects with type 2 diabetes inadequately controlled with metformin who attended our outpatient clinic for the entire period of observation, had never discontinued therapy, had never had side or adverse effects, had normal renal function (estimated glomerular filtration rate over $60 \mathrm{~mL} / \mathrm{min}$ ), modified their dietary habits according to our advices, and since baseline, underwent all blood tests in our outpatient clinic laboratory. If they were not able to perform self-blood glucose testing at home or did not modify according to our advices, then they were excluded. In patients whose $\mathrm{HbA}_{1 \mathrm{c}}$ level was $\geq 7.5 \%$, insulin or glimepiride were added to the treatment regimen at the same time of initiation of dapagliflozin (DAPA + MET + other glucose-lowering drugs), as per normal clinical practice and clinician's decision.

The ethics committee (Comitato Bioetico Palermo) at the investigational site was notified of the study protocol and the study was conducted in accordance with the Italian law and the Declaration of Helsinki.

2.2. Assessments. Baseline characteristics included age, sex, weight, waist circumference, systolic blood pressure, diastolic blood pressure, and disease duration.

Effectiveness was assessed using the change from baseline in $\mathrm{HbA}_{1 \mathrm{c}}$ at 6 and 12 months as well as changes in body mass index (BMI) and lipid parameters (total cholesterol, low density lipoprotein (LDL) cholesterol, high density lipoprotein (HDL) cholesterol, and triglycerides) at 12 months. $\mathrm{HbA}_{1 \mathrm{c}}$ was self-monitored, with outpatient visits performed every three months or when needed. Renal function was assessed by the change in blood creatinine levels at 6 and 12 months, as well as urine microalbumin levels at 12 months.

2.3. Statistical Analyses. Statistical analyses were performed using SPSS Statistics version 21 for Windows. All variables were analyzed by summary statistical methods and the analyses were performed separately in patients who received DAPA + MET and DAPA + MET + other glucose-lowering drugs. For continuous/quantitative variables, descriptive statistics, including the number of available values, arithmetic mean, standard deviation (SD), minimum, median, and maximum were calculated, while for categorical/qualitative variables, frequency tables were generated. Paired sample $t$-tests were performed using an analysis of covariance (ANCOVA) model with baseline values as a covariate with a significance level set at 5\%. General linear models were used to adjust for variation between patients who received $\mathrm{DAPA}+\mathrm{MET}$ and those who received DAPA + MET + other glucose-lowering drugs. Baseline values, age, and disease duration served as covariates, while sex served as the fixed factor.

\section{Results}

Data on 66 patients (mean age 56 years; 39\% female) were included in the study after database analysis of 235 type 2 diabetic subjects as reported in the Methods. At baseline, mean $\mathrm{HbA}_{1 \mathrm{c}}$ of the entire patient population was 9.2\%, while mean eGFR was $95.5 \mathrm{~mL} / \mathrm{min}$. Concomitant therapies included angiotensin-converting-enzyme inhibitors, angiotensin receptor blockers and calcium channel blockers for hypertension, and statins for management of dyslipidemia. The baseline demographics and characteristics were not significantly different between patients who received DAPA + MET alone and those who were prescribed additional therapy (DAPA + MET + other glucose-lowering drugs), with the exception of waist circumference and disease duration (Table 1). After 12 months of therapy, 29 (44\%) patients had initiated treatment with an additional glucose-lowering agent (glimepiride $2-4 \mathrm{mg} /$ day or insulin) and $12(18 \%)$ patients had discontinued metformin due to intolerance.

3.1. Effectiveness. When added to metformin, dapagliflozin significantly reduced $\mathrm{HbA}_{1 \mathrm{c}}$ levels from baseline in patients who received DAPA + MET, as well as in those who received $\mathrm{DAPA}+\mathrm{MET}+$ other glucose-lowering drugs (Figure 1). Significant reductions were observed after 6 and 12 months of treatment. Moreover, in all patients significant reductions were observed at 12 months compared with the $\mathrm{HbA}_{1 \mathrm{c}}$ levels at 6 months (Figure 1(b)). After 6 months of treatment, $\mathrm{HbA}_{1 \mathrm{c}}$ levels decreased by $1.4 \%$ (95\% confidence interval (CI): $1.1 \%$ to $1.7 \% ; p<0.001)$ and $0.8 \%$ (95\% CI: $0.5 \%$ to $1.1 \% ; p<0.001)$ in patients who received DAPA + MET and those who also received other glucose-lowering drugs, respectively. After 12 months of treatment, $\mathrm{HbA}_{1 \mathrm{c}}$ levels decreased by $1.7 \%$ (95\% CI: $1.5 \%$ to $2.0 \%$; $p<0.001$ ) and $1.3 \%$ (95\% CI $1.0 \%$ to $1.6 \% ; p<0.001)$, respectively. In patients who received DAPA + MET, the change from baseline in $\mathrm{HbA}_{1 \mathrm{c}}$ levels at 6 months was significantly greater that in patients who received DAPA + MET + other glucoselowering drugs $(p=0.020)$; however, no significant between-group difference was observed at 12 months $(p=0.053)$. Over the 12-month treatment period, dapagliflozin significantly reduced BMI in patients who received $\mathrm{DAPA}+\operatorname{MET}\left(\triangle \mathrm{BMI}=-0.7 \mathrm{~kg} / \mathrm{m}^{2}, 95 \% \mathrm{CI}:-1.1\right.$ to -0.3 ; $p<0.01)$ and DAPA + MET + other glucose-lowering drugs $\left(\Delta \mathrm{BMI}=-0.9 \mathrm{~kg} / \mathrm{m}^{2}, 95 \% \mathrm{CI}:-1.3\right.$ to $\left.-0.5, p<0.001\right)$ (Table 2), with no significant difference between patients. No significant changes in lipid parameters over the study period were observed.

3.2. Renal Function. No detrimental effects on renal function were observed during the study. There were no significant changes from baseline in creatinine levels at 6 and 12 months in all patients (Figure 2). On the other hand, after 12 months, a significant reduction in microalbumin was observed in patients who received DAPA + MET alone (Table 2). The 
TABLE 1: Baseline demographics and characteristics.

\begin{tabular}{lccc}
\hline Characteristic & DAPA + MET alone $(n=37)$ & $\begin{array}{c}\text { DAPA + MET + other glucose-lowering drugs } \\
(n=29)^{\mathrm{a}}\end{array}$ & $\begin{array}{c}\text { Total } \\
(n=66)\end{array}$ \\
\hline Age (years) & $57.1 \pm 9.3$ & $55.1 \pm 8.6$ & $56.3 \pm 9.0$ \\
Female, $n(\%)$ & $14(37.8)$ & $12(41.4)$ & $26(39.4)$ \\
Weight $(\mathrm{kg})$ & $91.6 \pm 17.9$ & $94.6 \pm 17.6$ & $93.0 \pm 17.7$ \\
Waist circumference $(\mathrm{cm})$ & $111.0 \pm 15.0$ & $119.0 \pm 16.2^{*}$ & $114.5 \pm 15.9$ \\
Disease duration (years) & $8.1 \pm 5.0$ & $11.6 \pm 8.5^{*}$ & $9.7 \pm 6.9$ \\
Systolic BP $(\mathrm{mmHg})$ & $138.6 \pm 18.5$ & $134.7 \pm 14.0$ & $136.8 \pm 16.6$ \\
Diastolic BP $(\mathrm{mmHg})$ & $81.5 \pm 9.3$ & $81.0 \pm 12.6$ & $81.3 \pm 10.8$ \\
HbA $(\%)$ & $8.8 \pm 1.6$ & $9.7 \pm 1.5$ & $9.2 \pm 1.6$ \\
BMI $\left(\mathrm{kg} / \mathrm{m}^{2}\right)$ & $33.1 \pm 6.2$ & $33.8 \pm 6.3$ & $33.4 \pm 6.1$ \\
Creatinine $(\mu \mathrm{mol} / \mathrm{L})$ & $75.1 \pm 17.6$ & $76.3 \pm 15.5$ & $75.6 \pm 18.2$ \\
Microalbumin $(\mu \mathrm{g} / \mathrm{mg})$ & $119.5 \pm 139.6$ & $67.1 \pm 80.6$ & $96.5 \pm 118.6$ \\
eGFR $(\mathrm{mL} / \mathrm{min})$ & $95.9 \pm 29.1$ & $96.6 \pm 24.1$ & $95.5 \pm 29.7$ \\
\hline
\end{tabular}

All values are presented as mean \pm standard deviation unless otherwise stated. BMI, body mass index; BP, blood pressure; DAPA, dapagliflozin; eGFR, estimated

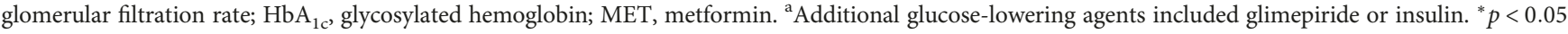
versus DAPA + MET alone.

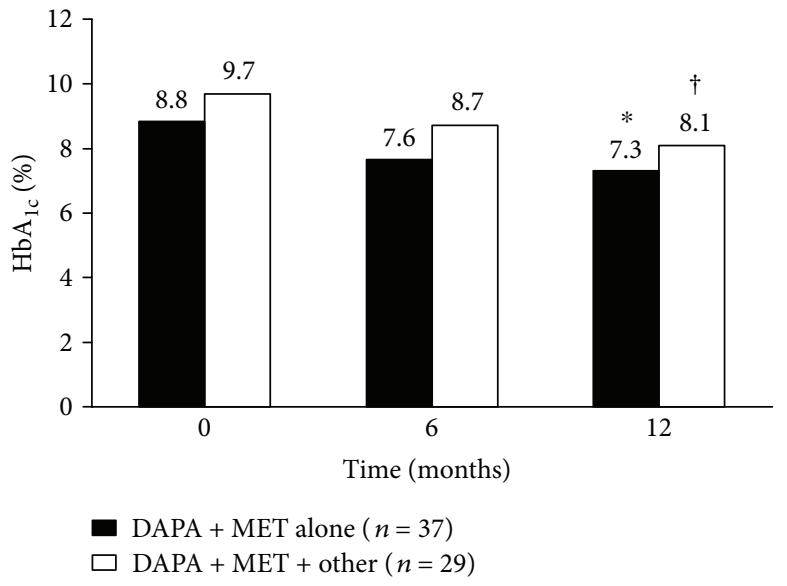

(a)

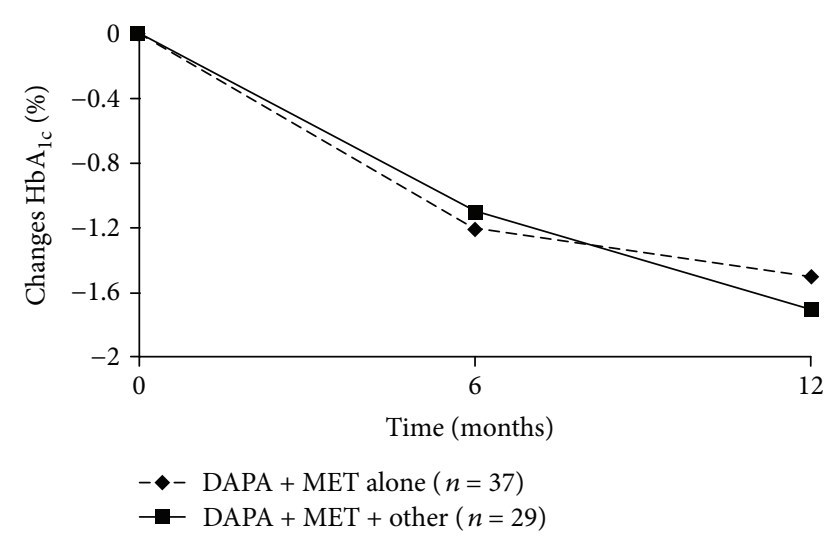

(b)

Figure 1: Glycosylated hemoglobin $\left(\mathrm{HbA}_{1 \mathrm{c}}\right)$ (a) at each study visit and (b) change from baseline. DAPA, dapagliflozin; MET, metformin; other, glimepiride or insulin. ${ }^{*} p<0.001$ DAPA + MET versus baseline; ${ }^{\dagger} p<0.001$ DAPA + MET + other glucose-lowering drugs versus baseline.

eGFR values in all patients remained above $60 \mathrm{~mL} / \mathrm{min}$ throughout the study, in accordance with the prescribing rules of the Italian Medicines Agency (AIFA). No statistically significant variation in eGFR was observed between treatment groups.

\section{Conclusions}

This retrospective observational study investigated the effectiveness and safety of add-on therapy with dapagliflozin in patients with type 2 diabetes treated at a single facility in Italy. The results demonstrate that dapagliflozin is effective and safe. Dapagliflozin significantly reduced $\mathrm{HbA}_{1 \mathrm{c}}$ levels after 12 months of treatment regardless of whether other glucose-lowering agents were received. In both patients, significant reductions were apparent from 6 months. Contrary to expectations, greater reductions were obtained in patients who received DAPA + MET, that is, those who received no additional therapy. We did not find any negative effects on renal function (assessed by blood creatinine and urine microalbumin levels) during the study. Microalbumin reduction was observed in patients who received DAPA + MET alone but we cannot exclude, due the nature of our study, other potential factors that could explain this exclusive effect.

The results of this study are in line with those of other studies of dapagliflozin conducted in patients with type 2 diabetes inadequately controlled with metformin [8-16].

This study had a number of limitations due to its retrospective observational design. It used data from an unselected 
TABLE 2: Secondary efficacy and safety parameters.

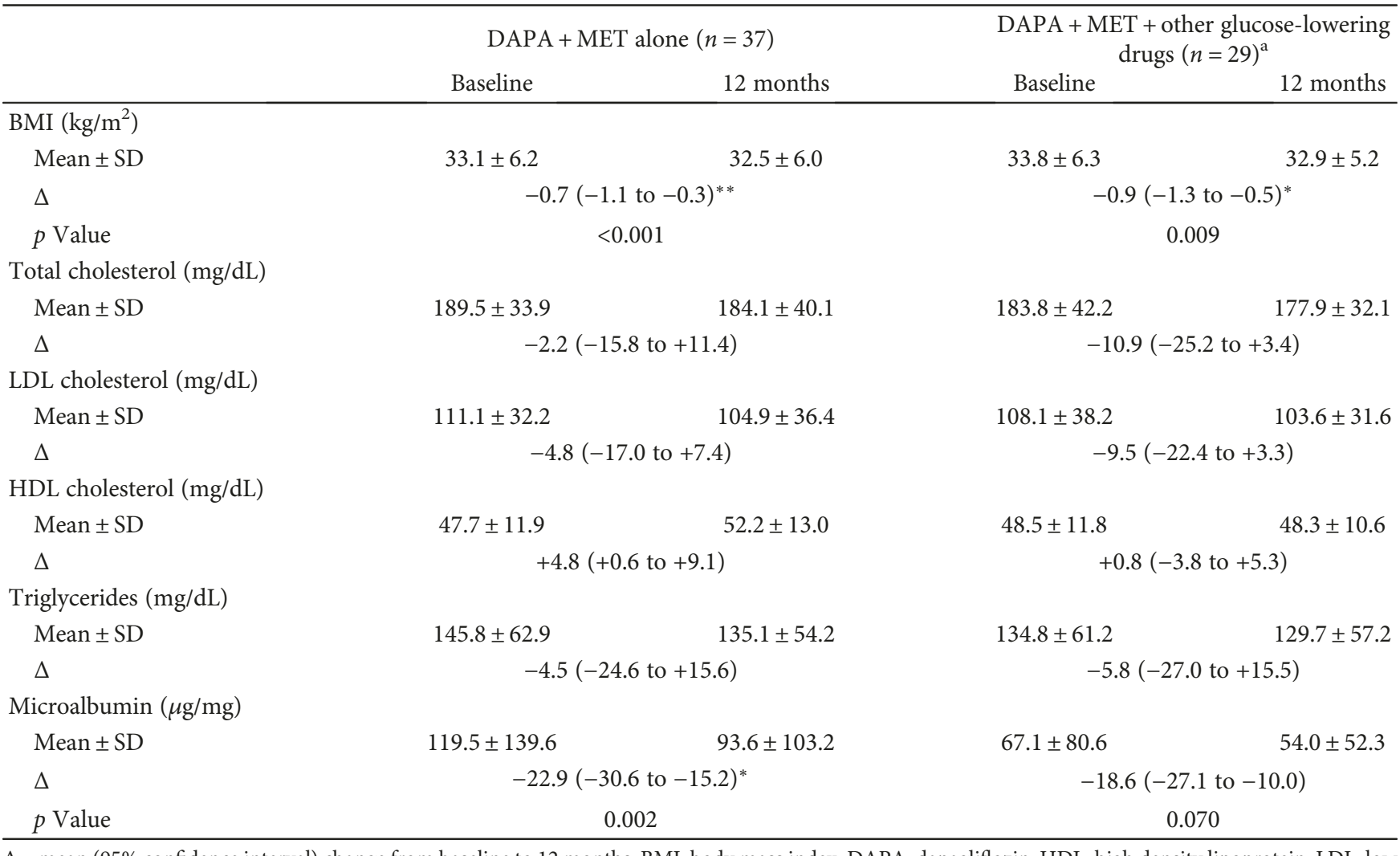

$\Delta=$ mean (95\% confidence interval) change from baseline to 12 months. BMI, body mass index; DAPA, dapagliflozin; HDL, high density lipoprotein; LDL, low density lipoprotein; MET, metformin; SD, standard deviation. ${ }^{a}$ Additional glucose-lowering agents included glimepiride or insulin. ${ }^{*} p<0.001$ and ${ }^{* *} p<0.01$.

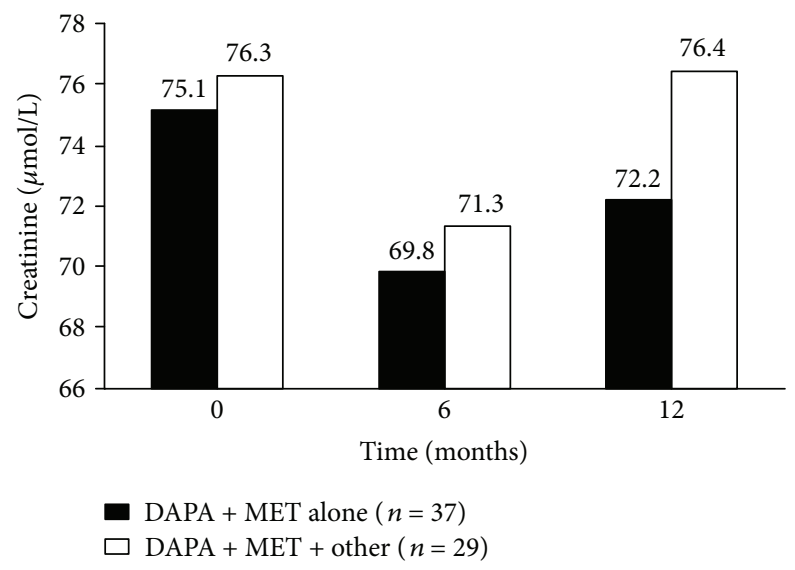

(a)

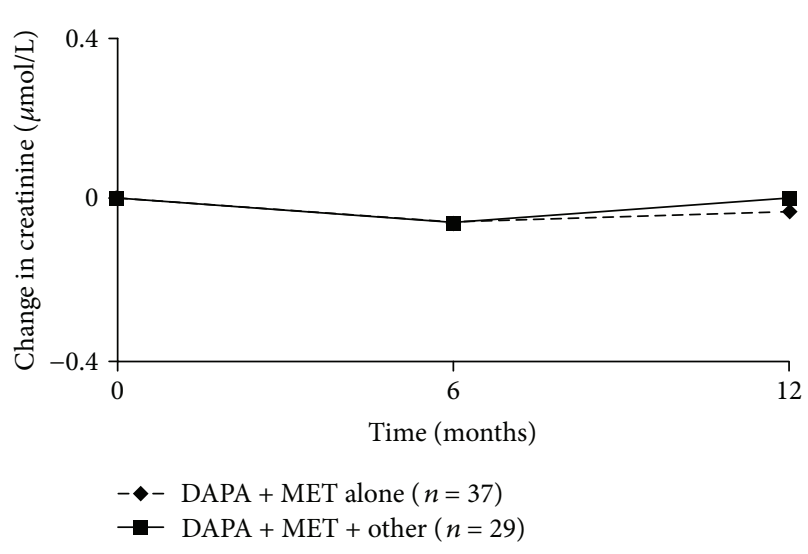

(b)

Figure 2: Creatinine (a) at each study visit and (b) change from baseline. DAPA, dapagliflozin; MET, metformin; other, glimepiride or insulin.

group of patients and the size of the patient population was not as large as in other studies conducted in patients with type 2 diabetes. Furthermore, no measures were taken to control for confounding factors. This, in combination with a lack of a control group, means that this study cannot adequately explain the finding that patients treated with DAPA + MET experienced greater reductions in $\mathrm{HbA1c}$ levels compared with those who received additional glucose-lowering drugs. The strengths of this study, on the other hand, included the length of the observational period and the fact that it was conducted in a real-life setting. These factors improve the generalizability of its findings. In conclusion, this 
retrospective observational study demonstrates that dapagliflozin is effective in reducing $\mathrm{HbA}_{1 \mathrm{c}}$ levels and safe when administered in a real-life setting in Italy in patients with type 2 diabetes also receiving metformin. Glycemic control was more likely to be achieved in patients treated with dapagliflozin alone or in combination with metformin, while add-on therapy with insulin and/or glimepiride was not associated with further improvement in $\mathrm{HbA}_{1 \mathrm{c}}$ levels.

\section{Conflicts of Interest}

The authors declare that there are no conflicts of interest regarding the publication of this paper.

\section{Acknowledgments}

The authors would like to thank Georgii Filatov of Springer Healthcare Communications for the preparation of the manuscript up to submission, and Valentina Mirisola of Mediservice (Italy) who provided statistical analysis of the data. Sponsorship for medical writing assistance, statistical support, and the article processing charges for this publication were funded by AstraZeneca (Italy).

\section{References}

[1] O. Disoteo, F. Grimaldi, E. Papini et al., "State-of-the-art review on diabetes care in Italy," Annals of Global Health, vol. 81, no. 6, pp. 803-813, 2015.

[2] S. E. Inzucchi, R. M. Bergenstal, J. B. Buse et al., "Management of hyperglycemia in type 2 diabetes, 2015: a patient-centered approach: update to a position statement of the American Diabetes Association and the European Association for the Study of Diabetes," Diabetes Care, vol. 38, no. 1, pp. 140-149, 2015.

[3] M. Stumvoll, B. J. Goldstein, and T. W. van Haeften, "Type 2 diabetes: principles of pathogenesis and therapy," The Lancet, vol. 365, no. 9467, pp. 1333-1346, 2005.

[4] R. de Marco, F. Locatelli, G. Zoppini, G. Verlato, E. Bonora, and M. Muggeo, "Cause-specific mortality in type 2 diabetes. The Verona Diabetes Study," Diabetes Care, vol. 22, no. 5, pp. 756-761, 1999.

[5] Y. Kanai, W. S. Lee, G. You, D. Brown, and M. A. Hediger, "The human kidney low affinity $\mathrm{Na}^{+} /$glucose cotransporter SGLT2. Delineation of the major renal reabsorptive mechanism for D-glucose," The Journal of Clinical Investigation, vol. 93, no. 1, pp. 397-404, 1994.

[6] J. F. List, V. Woo, E. Morales, W. Tang, and F. T. Fiedorek, "Sodium-glucose cotransport inhibition with dapagliflozin in type 2 diabetes," Diabetes Care, vol. 32, no. 4, pp. 650-657, 2009.

[7] E. Ferrannini, S. J. Ramos, A. Salsali, W. Tang, and J. F. List, "Dapagliflozin monotherapy in type 2 diabetic patients with inadequate glycemic control by diet and exercise: a randomized, double-blind, placebo-controlled, phase 3 trial," Diabetes Care, vol. 33, no. 10, pp. 2217-2224, 2010.

[8] C. J. Bailey, J. L. Gross, D. Hennicken, N. Iqbal, T. A. Mansfield, and J. F. List, "Dapagliflozin add-on to metformin in type 2 diabetes inadequately controlled with metformin: a randomized, double-blind, placebo-controlled 102-week trial," BMC Medicine, vol. 11, no. 1, p. 43, 2013.
[9] C. J. Bailey, J. L. Gross, A. Pieters, A. Bastien, and J. F. List, "Effect of dapagliflozin in patients with type 2 diabetes who have inadequate glycaemic control with metformin: a randomised, double-blind, placebo-controlled trial," The Lancet, vol. 375, no. 9733, pp. 2223-2233, 2010.

[10] S. Del Prato, M. Nauck, S. Duran-Garcia et al., "Long-term glycaemic response and tolerability of dapagliflozin versus a sulphonylurea as add-on therapy to metformin in patients with type 2 diabetes: 4-year data," Diabetes, Obesity and Metabolism, vol. 17, no. 6, pp. 581-590, 2015.

[11] M. A. Nauck, S. Del Prato, J. J. Meier et al., "Dapagliflozin versus glipizide as add-on therapy in patients with type 2 diabetes who have inadequate glycemic control with metformin: a randomized, 52-week, double-blind, active-controlled noninferiority trial," Diabetes Care, vol. 34, no. 9, pp. 2015-2022, 2011.

[12] M. A. Nauck, S. Del Prato, S. Duran-Garcia et al., "Durability of glycaemic efficacy over 2 years with dapagliflozin versus glipizide as add-on therapies in patients whose type 2 diabetes mellitus is inadequately controlled with metformin," Diabetes, Obesity and Metabolism, vol. 16, no. 11, pp. 1111-1120, 2014.

[13] L. A. Leiter, W. T. Cefalu, T. W. de Bruin, I. Gause-Nilsson, J. Sugg, and S. J. Parikh, "Dapagliflozin added to usual care in individuals with type 2 diabetes mellitus with preexisting cardiovascular disease: a 24-week, multicenter, randomized, double-blind, placebo-controlled study with a 28-week extension," Journal of the American Geriatrics Society, vol. 62, no. 7, pp. 1252-1262, 2014.

[14] W. T. Cefalu, L. A. Leiter, T. W. de Bruin, I. Gause-Nilsson, J. Sugg, and S. J. Parikh, "Dapagliflozin's effects on glycemia and cardiovascular risk factors in high-risk patients with type 2 diabetes: a 24-week, multicenter, randomized, double-blind, placebo-controlled study with a 28 -week extension," Diabetes Care, vol. 38, no. 7, pp. 1218-1227, 2015.

[15] T. Nystrom, J. Bodegard, D. Nathanson, M. Thuresson, A. Norhammar, and J. W. Eriksson, "Novel oral glucoselowering drugs are associated with lower risk of all-cause mortality, cardiovascular events and severe hypoglycaemia compared with insulin in patients with type 2 diabetes," Diabetes, Obesity and Metabolism, vol. 19, no. 6, pp. 831-841, 2017.

[16] K. A. Toulis, B. H. Willis, T. Marshall et al., "All-cause mortality in patients with diabetes under treatment with dapagliflozin: a population-based, open-cohort study in the health improvement network database," The Journal of Clinical Endocrinology \& Metabolism, vol. 102, no. 5, pp. 1719-1725, 2017. 


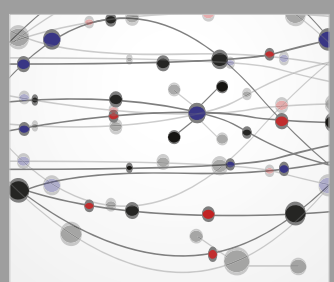

The Scientific World Journal
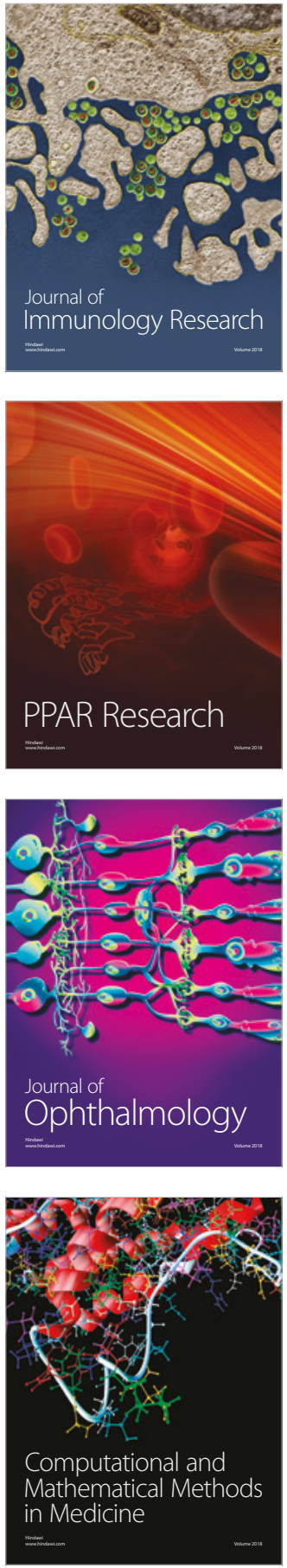

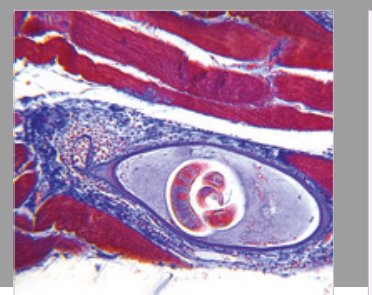

Gastroenterology Research and Practice

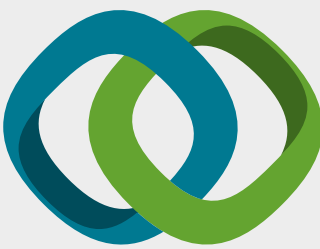

\section{Hindawi}

Submit your manuscripts at

www.hindawi.com
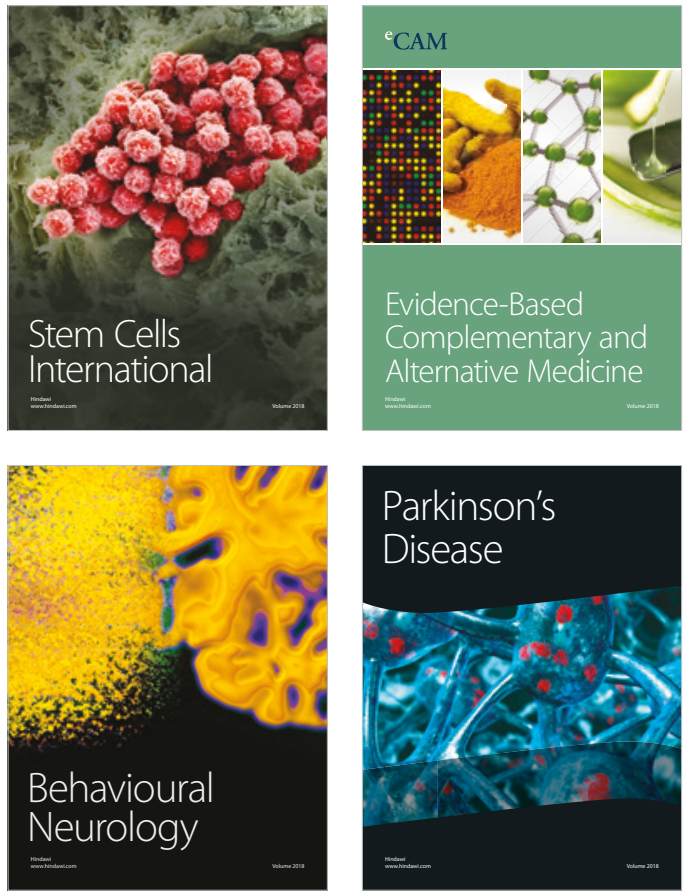

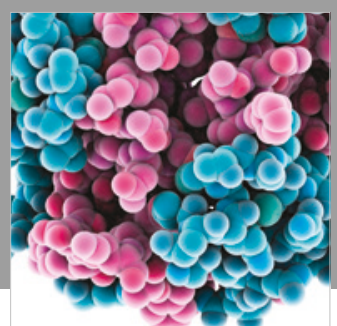

ournal of

Diabetes Research

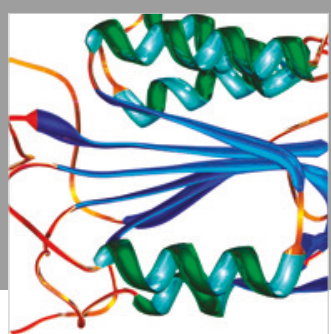

Disease Markers
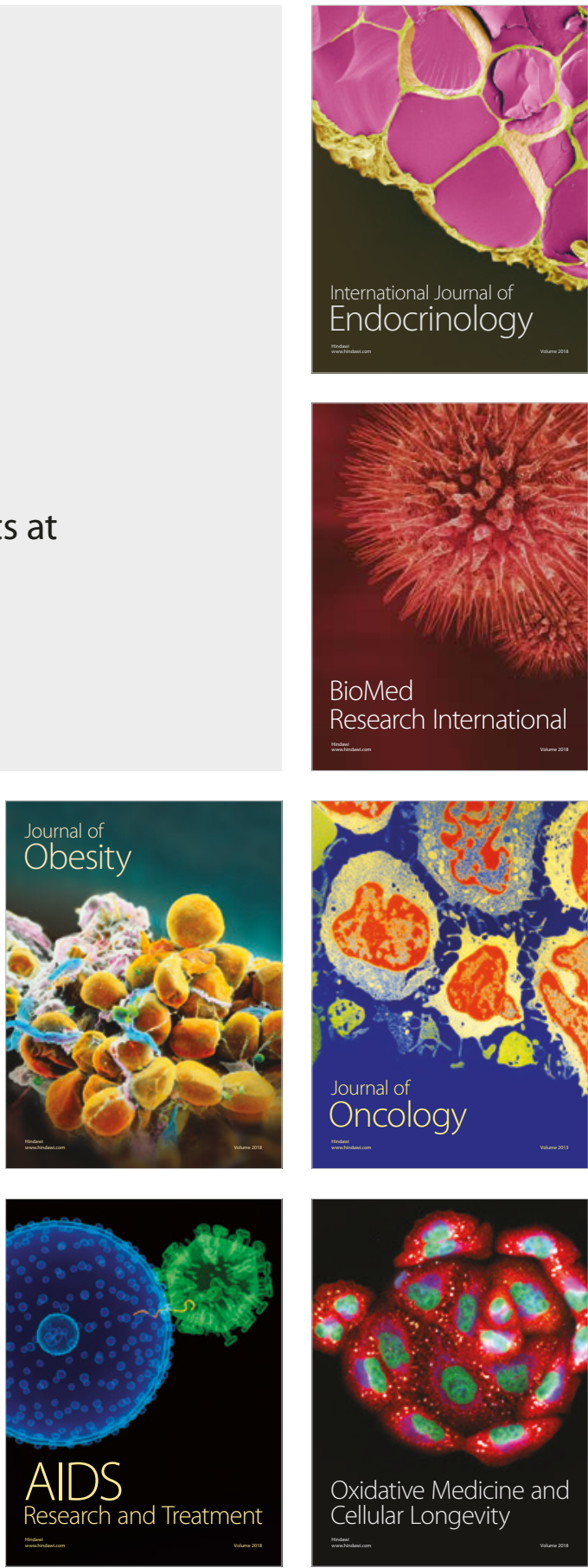